\title{
Longitudinal Relaxation Rate
}

National Cancer Institute

\section{Source}

National Cancer Institute. Longitudinal Relaxation Rate. NCI Thesaurus. Code C80361.

The relaxation rate of the net nuclear spin polarization in the direction of the magnetic field of a spectrometer back to its equilibrium value. 